\title{
GERENCIA DO CUIDADO DE ENFERMAGEM NA HEMOTERAPIA EM SERVIÇO HOSPITALAR DE ENFERMAGEM
}

\author{
MANAGEMENT OF NURSING \\ CARE IN HEMOTHERAPY IN A \\ HOSPITAL NURSING SERVICE
}

\section{GESTIÓN DE LOS CUIDADOS DE ENFERMERÍA EN HEMOTERAPIA EN UN SERVICIO DE ENFERMERÍA HOSPITALARIA}

\author{
Hilma Nazaré Mendes Bezerra ${ }^{1}$ \\ Aurilívia Carolinne Lima Barros ${ }^{2}$ \\ Rosana Amora Ascari ${ }^{3}$ \\ Thaís Cristina Flexa Souza ${ }^{4}$ \\ Jouhanna do Carmo Menegaz ${ }^{5}$
}

Como citar este artigo: Bezerra HNM, Barros ACL, Ascari RA, Souza TCF, Menegaz JC. Gerência do cuidado de enfermagem na hemoterapia em serviço hospitalar de enfermagem. Rev baiana enferm. 2022;36:e45076.

Objetivo: analisar a gerência do cuidado de Enfermagem na hemoterapia em um hospital universitário. Método: estudo descritivo, exploratório de abordagem qualitativa, realizado com 29 enfermeiros de Hospital Universitário de Belém, Pará, Brasil. Foram aplicadas entrevistas semiestruturadas entre agosto/2018 e janeiro/2019. Os dados coletados foram submetidos à análise de conteúdo temática. Resultados: Coordenação do cuidado/Supervisão e Prestação do cuidado complexo foram as ações mais mencionadas quanto aos procedimentos hemoterápicos. Dimensionamento da equipe, Gestão de materiais, Planejamento do cuidado de enfermagem e Avaliação dos resultados das ações de enfermagem foram ações ausentes nas falas dos enfermeiros. Treinamento/educação da equipe mostrou-se fragilizado. Já Liderança não foi mencionada explicitamente, mas pôde estar presente, ainda que de forma fragilizada. Considerações finais: as ações mais representadas nas falas dos enfermeiros estavam relacionadas às competências técnicas adquiridas no contexto formativo, enquanto as ações menos mencionadas relacionaram-se às competências e habilidades que comumente permeiam lacunas da educação formal.

Descritores: Enfermagem. Cuidados de Enfermagem. Serviço Hospitalar de Enfermagem. Serviço de Hemoterapia. Gestão em Saúde.

Objective: analyzing the management of nursing care in hemotherapy in a university hospital. Method: a descriptive, exploratory study with a qualitative approach conducted with 29 nurses from the University Hospital of Belém, Pará,

\footnotetext{
Enfermeira. Mestre em Enfermagem. Enfermeira da Agência Transfusional do Hospital Universitário João de Barros Barreto. Belém, Pará, Brasil. hilmambezerra@gmail.com. https://orcid.org/0000-0002-7987-3।40.

Enfermeira. Mestre em Enfermagem. Pesquisadora Independente. Belém, Pará, Brasil. aurilivia.barros@gmail.com. https://orcid.org/0000-0002-4096-0546.

Enfermeira. Doutora em Enfermagem. Professora do Programa de Pós-Graduação em Enfermagem da Universidade do Estado de Santa Catarina. Chapecó, Santa Catarina, Brasil. https://orcid.org/0000-0002-2281-8642.

Enfermeira. Mestre em Enfermagem. Pesquisadora Independente. Belém, Pará, Brasil. https://orcid.org/0000-0002-7296-0380.

Enfermeira. Doutora em Enfermagem. Professora do Departamento de Enfermagem da Universidade do Estado de Santa Catarina e do Programa de Pós-Graduação em Enfermagem da Universidade Federal do Pará. Chapecó, Santa Catarina, Brasil. https://orcid.org/0000-0002-7655-9826.
} 
Brazil. Semi-structured interviews were applied between August/2018 and January/2019. The collected data were submitted to thematic content analysis. Results: coordination of care/supervision and provision of complex care were the most mentioned actions regarding hemotherapy procedures. Team dimensioning, Materials management, Nursing care planning and Evaluation of the results of nursing actions were actions absent in the nurses' statements. Training/education of the team was weakened. Leadership was not explicitly mentioned, but it could be present, albeit in a fragile way. Final considerations: the actions most represented in the nurses' statements were related to the technical competencies acquired in the formative context, while the less mentioned actions were related to the competencies and skills that commonly permeate gaps in formal education.

Descriptors: Nursing. Nursing Care. Nursing Service. Hospital. Hemotherapy Service. Health Management.

Objetivo: analizar el manejo de los cuidados de enfermería en hemoterapia en un hospital universitario. Método: se trata de un estudio descriptivo, exploratorio con enfoque cualitativo realizado con 29 enfermeras del Hospital Universitario de Belém, Pará, Brasil. Se aplicaron entrevistas semiestructuradas entre agosto/2018 y enero/2019. Los datos recopilados se sometieron a análisis de contenido temático. Resultados: la coordinación de la atención/ supervisión y la prestación de cuidados complejos fueron las acciones más mencionadas con respecto a los procedimientos de hemoterapia. El dimensionamiento del equipo, la gestión de materiales, la planificación de los cuidados de enfermería y la evaluación de los resultados de las acciones de enfermería fueron acciones ausentes en las declaraciones de las enfermeras. La capacitación/educación del equipo se debilitó. El liderazgo no se menciona explícitamente, pero podría estar presente, aunque de una manera frágil. Consideraciones finales: las acciones más representadas en las declaraciones de las enfermeras se relacionaron con las competencias técnicas adquiridas en el contexto formativo, mientras que las acciones menos mencionadas se relacionaron con las competencias y habilidades que comúnmente impregnan las brechas en la educación formal.

Descriptores: Enfermería. Atención de Enfermería. Servicio de Enfermería en Hospital. Servicio de Hemoterapia. Gestión en Salud.

\section{Introdução}

A hemoterapia é considerada como o emprego terapêutico do sangue em sua totalidade ou por meio de seus componentes e derivados, podendo ocorrer de forma programada, de rotina ou de urgência e emergência ${ }^{(1-2)}$. É inquestionável seu potencial para salvar vidas e contribuir para a estabilização de indivíduos em condições de instabilidade hemodinâmica, sendo considerada uma tecnologia moderna e relevante de terapêutica ${ }^{(1-3)}$.

O profissional médico é o responsável técnico pelo serviço de hemoterapia e a ele compete desde a indicação da transfusão até o cumprimento das normas técnicas. A equipe de enfermagem, por sua vez, possui forte atuação em todas as etapas do ciclo do sangue. Ao enfermeiro, compete ações desde a captação do sangue e de seus componentes até a transfusão e o descarte de resíduos. A importância desse profissional ocorre não apenas na execução técnica dos procedimentos, como também no planejamento e supervisão da equipe de enfermagem sob sua orientação ${ }^{(1-2)}$.

Considerada a última etapa da cadeia do sangue, em que o insumo é infundido no receptor, a técnica transfusional é um momento complexo. Deve-se considerar que toda transfusão pode gerar um incidente transfusional, necessitando, assim, de enfermeiros e de uma equipe multiprofissional com adequados conhecimentos para reconhecer e atender as intercorrências ${ }^{(4)}$.

Os conhecimentos e as habilidades de enfermagem adequados, utilizados oportunamente, são uma maneira eficiente de garantir a segurança do processo transfusional. Tal capacidade é fundamental para garantir a apropriada atuação profissional, bem como prevenir a ocorrência de situações indesejadas no processo terapêutico ${ }^{(5)}$. A atuação da enfermagem, responsável por promover uma assistência competente, resolutiva e segura, é, portanto, essencial para o sucesso desse processo ${ }^{(2)}$. Dessa forma, cabe ao enfermeiro manter-se vigilante para o surgimento de incidentes que possam porventura ocorrer durante o processo transfusional, para agir de forma atenta e imediata ${ }^{(4)}$.

Uma atribuição do enfermeiro, que se relaciona diretamente com a qualidade da ação de enfermagem, promovendo melhores 
condições de trabalho e de atuação, é a gerência do cuidado. Discutir a gerência do cuidado, nesse sentido, contribui para a ampliação das reflexões quanto à tríade cuidar-gerenciar-educar na perspectiva do cuidado de enfermagem ${ }^{(6)}$.

Em estudo relacionado à gerência do cuidado em enfermagem, pesquisadores elencaram oito ações, nas quais, por meio da descrição, ficou evidente a conexão e complementaridade entre assistência, gerência e educação para a práxis do cuidado em enfermagem, bem como sua importância na formação acadêmica e simultânea/posterior à aplicação prática. Este estudo aborda as seguintes ações de gerência do cuidado de enfermagem: Planejamento do cuidado de enfermagem; Prestação do cuidado complexo; Dimensionamento da equipe; Coordenação do cuidado/Supervisão; Treinamento/educação da equipe; Liderança; Gestão de materiais; e Avaliação dos resultados das ações de enfermagem ${ }^{(6)}$.

Considerando a gerência do cuidado de enfermagem no serviço de hemoterapia como essencial para a adequada e segura prática do ato transfusional, à luz das ações de Gerência do cuidado na prática profissional do enfermeiro acima elencadas ${ }^{(6)}$, questiona-se: Como ocorre a gerência do cuidado de enfermagem na hemoterapia? Assim, o objetivo deste artigo é analisar a gerência do cuidado de Enfermagem na hemoterapia em um hospital universitário.

\section{Método}

Estudo descritivo, analítico, de abordagem qualitativa. Esta investigação foi conduzida e estruturada com base nos Critérios de Consolidação para Relatórios de Pesquisa Qualitativa $(\text { COREQ })^{(7)}$. Foi realizada em um Complexo Hospitalar Universitário localizado na região Norte do Brasil.

O local de estudo foi um hospital de médio porte, referência estadual em Infectologia, Oncologia, Endocrinologia e Diabetes, gerido pela Empresa Brasileira de Serviços Hospitalares (EBSERH), que ofertava atendimento de média e alta complexidade. Possuía 108 enfermeiros em seu quadro profissional.
Para o estudo, foi destacada uma amostra intencional. Participaram 29 enfermeiros lotados nos setores de clínica médica, infectologia e terapia intensiva, por serem as que mais utilizam o serviço hemoterápico. Como critérios de inclusão, considerou-se ser enfermeiro, com vínculo funcional, lotado na unidade em estudo, atuando na assistência direta. Excluíram-se enfermeiros cedidos temporariamente para as unidades hospitalares investigadas, profissionais em férias ou licenciados por qualquer motivo durante o período de coleta de dados.

Aprovado o protocolo de pesquisa, a primeira autora contatou a supervisão geral de enfermagem do citado Hospital, para dar ciência da pesquisa e obter permissão para iniciar levantamento de dados. Em um segundo momento, essa mesma autora apresentou-se nas clínicas, solicitou participação dos enfermeiros nos três turnos de trabalho e forneceu informações sobre o objetivo da pesquisa.

A coleta de dados, por meio de entrevista com roteiro semiestruturado, ocorreu entre agosto de 2018 e janeiro de 2019, com agendamento prévio, de acordo com a disponibilidade dos participantes. Foi realizada em locais privados no próprio local de trabalho, após assinatura do Termo de Consentimento Livre e Esclarecido (TCLE) e do Termo de Gravação, com duração entre 4 e 21 minutos. As entrevistas foram audiogravadas em um aparelho de telefone celular, com autorização prévia, e posteriormente transcritas para documento em Word e enviadas aos participantes, para validação do conteúdo.

No momento da coleta, buscou-se identificar as dificuldades e limitações operacionais do enfermeiro, que pudessem interferir na qualidade do atendimento hemoterápico. Dessa forma, pôde-se explorar o conhecimento e a percepção do profissional quanto ao exercício da supervisão e, nesse sentido, voltou-se essencialmente para questões gerenciais.

O roteiro de entrevista continha variáveis de caracterização, tais como: sexo, formação profissional, vínculo empregatício, formas de acesso ao conhecimento sobre hemoterapia e legislações regulamentadoras da prática na vida 
acadêmica e profissional. Foi realizado um teste piloto em julho de 2018, que confirmou a adequação do instrumento para a investigação.

Após a validação do conteúdo das entrevistas pelos participantes, os dados foram analisados por meio da técnica de análise de conteúdo ${ }^{(8)}$. As etapas de pré-análise, descrição analítica e tratamento dos resultados, inferência e interpretação possibilitaram a identificação de três categorias, a saber: Percepção sobre o processo de trabalho do enfermeiro na instituição, Conhecimento técnico do enfermeiro sobre hemoterapia e Gerência do cuidado de enfermagem em hemoterapia.

Neste artigo, são apresentados, de forma articulada, os achados relativos à primeira e à terceira categorias, discutidos à luz das ações de Gerência do cuidado na prática profissional do enfermeiro: Planejamento do cuidado de enfermagem, Prestação do cuidado complexo, Dimensionamento da equipe, Coordenação do cuidado/Supervisão, Treinamento/educação da equipe, Liderança, Gestão de materiais, e Avaliação dos resultados das ações de enfermagem ${ }^{(6)}$.

Assim, serão discutidas as ações de Gerência do Cuidado presentes nas práticas de gerência do cuidado de enfermagem em hemoterapia e ações de Gerência do Cuidado ausentes ou fragilizadas nas práticas de gerência do cuidado de enfermagem na hemoterapia.

O estudo seguiu as recomendações da Resolução $n^{\circ} 466 / 2016$, do Conselho Nacional de Saúde, e foi aprovado pelo Comitê de Ética em Pesquisa do Hospital Universitário João de Barros Barreto, Parecer n⿳o 2.165.945/2018. O anonimato dos participantes foi preservado por meio da utilização da sigla "ENF", de Enfermeiro participante, seguida de número arábico crescente, de acordo com a ordem de participação no estudo.

\section{Resultados}

Participaram deste estudo, 29 enfermeiros com idade entre 26 e 55 anos, prevalecendo a faixa etária de 41 a $45 \operatorname{anos}(24 \%)$ e sexo feminino (69\%). Quanto ao tempo de formação, os profissionais mais antigos tinham entre $30 \mathrm{e}$ 35 anos (21\%) e os mais novos, até cinco anos de formação (7\%). Mais de um quarto dos participantes (27\%) sinalizaram tempo de atuação entre 6 e 10 anos, mesmo percentual de profissionais atuantes há mais de 20 anos. Do total de participantes, 15 referiram ser servidores da EBSERH (52\%) e 14 eram da Universidade (48\%). Mais da metade deles (62\%) apresentava apenas um vínculo empregatício.

Quanto à Instituição de Formação, 19 (65\%) eram egressos de instituições públicas de ensino superior, sendo 11 (38\%) de instituições estaduais e 8 (28\%) de instituições federais.

Conforme a análise de conteúdo das entrevistas realizadas, identificou-se, em relação às Práticas de Gerência do Cuidado em Hemoterapia, que os participantes afirmaram realizar duas (Coordenação do cuidado/Supervisão e Prestação do cuidado complexo) das oito ações de gerência do cuidado investigadas no estudo, conforme apresentado a seguir.

\section{Ações de Gerência do Cuidado presentes nas práticas de gerência do cuidado de enfermagem em hemoterapia}

Coordenação do cuidado/Supervisão foi a ação mais mencionada pelos enfermeiros, no que diz respeito aos procedimentos hemoterápicos. Entretanto, não se pode considerar que é uma ação de consenso, tendo em vista que alguns deles entendiam não haver padronização das condutas.

\footnotetext{
Bom, basicamente, o que nós fazemos, é supervisionar [...] os serviços de enfermagem [...] fazemos aprazamentos das medicações [...] as intercorrências que ocorrem durante o decorrer do plantão, onde a gente presta todas as nossas assistências [...] Eu acho que é isso. (ENF 2).

Sim. No caso de transfusão, a gente procura [...] é uma das prioridades né, quando o médico solicita a transfusão, então eu tenho logo que priorizar. Então, eu tenho que ver se esse paciente tem amostra ou não, tá? Isso eu solicito ao técnico, que ele colha amostra. Eu vou ver [...] ter o cuidado de [...] ver a identificação do pedido com a coleta, supervisionar para que o técnico, ele esteja identificando [...] o frasquinbo coletor, tá? Ele também tem que assinar a guia ou [...] a ficha [...] do pedido por ele coletado e encaminhar e esperar [...] (ENF 4).
}

Eu tenbo que olhar! Eu tenho que olbar, porque, se não tiver bem preenchido, principalmente [...] atrás a assina- 
tura do paciente autorizando [...] vai voltar do serviço. Então, a gente tem que fazer. (ENF 6).

Mas percebo que a gente não tem uma orientação única. Parece que, assim, tem várias pessoas, uma atirando para cada lado. Então, eu percebo, na nossa atividade diária, a falta de um padrão operacional [...] (ENF 14).

$\mathrm{Na}$ rotina dos serviços de hemoterapia, os enfermeiros mencionaram também a Prestação do cuidado complexo.

\begin{abstract}
Em sintese, as minhas atribuições aqui, como enfermeiro assistencial [...] resume-se em: prestação de cuidados diretos [...] aos pacientes, principalmente aos pacientes mais complexos tanto em terapia e quanto à prestação de cuidados mesmo [...] ferimentos e complexidade geral e [...] resume-se, em linhas gerais, nesses aspectos. (ENF 11).

Minha rotina, de modo geral, eu recebo [...] pego o plantão da colega, tanto do masculino quanto do feminino, depois [...] divido aqueles pacientes. A gente passa a visita, vê as situações do paciente, vê sonda, vê alimentação, vê a situação de curativo; quem tem curativo complexo, a gente arruma bandeja, espera o técnico dar banho e faz os curativos complexos. Depois a gente vai vendo as intercorrências, geralmente a escala é por complexidade, demanda também um pouco de tempo pra gente fazer, e depois a gente vai fazer a evolução desses pacientes. E aí, no decorrer do plantão, vem o aprazamento de medicações, problemas para serem resolvidos na farmácia, transfusôes de sangue que chegam. Interação com a equipe multidisciplinar é rotina [...] são várias rotinas. (ENF 6).
\end{abstract}

\section{Ações de Gerência do Cuidado ausentes ou fragilizadas nas práticas de gerência do cuidado de enfermagem na bemoterapia}

Aspectos do Dimensionamento da equipe e Gestão de materiais não foram citados nas falas dos enfermeiros, assim como foi ausente a menção ao Planejamento do cuidado de enfermagem e Avaliação dos resultados das ações de enfermagem. Liderança não foi mencionada de forma explícita, mas pode estar sendo exercida de forma fragilizada. Quando destacada, não foi na perspectiva de influência, e sim de demarcação de atribuições, sugerindo que se adota um estilo centrado nas tarefas e não nas pessoas.

\footnotetext{
Eu acho que a legislação já coloca que é atribuição do enfermeiro. Quando o enfermeiro coloca isso para outra pessoa, é difícil para mim mudar uma rotina de uma instituição. Quando você delega uma atribuição sua, você perde o seu espaço. (ENF 6).

Eu entendo que todo o processo é exclusivamente do enfermeiro. Então, eu, de outras rotinas de bospital [...] se o meu paciente vai fazer uma transfusão, eu gosto de receber a bolsa, de conferir a tipagem sanguínea da bolsa, juntamente com oformulário da agência. (ENF 12).
}

O Treinamento/educação da equipe parece também fragilizado pela própria dificuldade dos enfermeiros quanto a conhecimentos sobre hemoterapia. Pôde-se perceber a insegurança desses profissionais com a coordenação do cuidado, por não terem recebido treinamento.

\section{Treinamento mesmo, nunca tive. (ENF 14).}

Eu não tive acesso e também estou aprofundando nesse assunto, para saber se existe um protocolo padrão de bemotransfusão, do passo a passo, das possíveis identificações de reações transfusional [...] (ENF 11)

Treinamento. Pode ser até em serviço. Treinamentos em serviço para toda a equipe: enfermeiros, técnicos. (ENF 16).

Várias vezes, chegou-se a apontar a necessidade de produção de material, como a elaboração de protocolos e manuais de procedimentos padrão para a hemoterapia.

\begin{abstract}
Eu sugiro um protocolo de instruções técnicas de como proceder um fluxograma, de como vai cada etapa, de como se procede, treinamentos com a equipe. Com relação a isso, que venha alguém, converse com a gente como funciona, como é que vai, quando eu precisar de sangue, como é que eu faço, quem é que assina, quem recebe, quem não recebe. (ENF 19).
\end{abstract}

Eu acho que deveria ter um trabalho voltado mais especificamente pra conscientização deles, dos perigos da transfusão, que pode acontecer reações, porque eles não estão cientes disso. (ENF10).

Educação continuada com os funcionários, e com a gente também, porque está sempre entrando coisas novas, sempre havendo mudanças na legislação e no cuidado a esse paciente. Eu acho que é importante o HEMOPA trazer para a gente. (ENF 24).

\section{Discussão}

No ambiente hospitalar, o exercício da função do enfermeiro no cuidado ao paciente crítico é de extrema importância e está intimamente ligado à Coordenação do cuidado/Supervisão ${ }^{(6)}$. Dessa forma, esse profissional assume responsabilidades para o desenvolvimento de tal função, que podem ser enfrentadas como um grande desafio. Para atender a essas demandas e direcionar as decisões estratégicas no ambiente de trabalho, os enfermeiros devem investir continuamente em seu crescimento pessoal e profissional, tendo em vista que o desempenho da função de coordenação requer conhecimentos e habilidades específicos para a melhor prestação do cuidado ${ }^{(5-6)}$. Neste sentido, a coordenação do 
cuidado é definida pela capacidade de integrar todo o cuidado que o paciente recebe em diferentes pontos, com o objetivo de prover cuidado continuado, por meio do gerenciamento entre os serviços ${ }^{(9)}$.

Outra prática evidenciada no contexto profissional dos gerentes de enfermagem atuantes no serviço de hemoterapia estudado foi a Prestação de cuidado complexo, em que o trabalho da equipe foi apresentado pelos participantes de forma diferenciada, por complexidade de procedimentos. Tal dinâmica justifica-se, pois a realização de procedimentos complexos de enfermagem é da competência exclusiva do enfermeiro, haja vista a sua formação incluir o preparo técnico adequado para o atendimento de tais necessidades de grupos restritos de clientes, que requerem cuidados críticos de saúde ${ }^{(2)}$.

Sabe-se que existem, na literatura disponibilizada tanto pelo Conselho Federal de Enfermagem $(\mathrm{COFEN})^{(2,10)}$ como pelo Ministério da Saúde ${ }^{(3,11-12)}$, por meio de normas regulamentadoras, marcos conceituais, guias, protocolos, portarias e manuais, diferentes informações que envolvem a gerência do cuidado esperada do profissional enfermeiro.

A evidência apresentada neste estudo, ao identificar o descompasso entre a formação e a prática do enfermeiro, apenas reitera os achados de estudo sobre as práticas de enfermeiros na gerência do cuidado em enfermagem e saúde ${ }^{(6)}$, em que, muitas vezes, a formação proporcionada não converge para a qualidade da assistência prestada. Ressalta-se que a fragilidade nos conhecimentos adquiridos, a baixa habilidade em sua implementação prática, bem como a dificuldade em agregar novos conhecimentos após o período de educação formal, geram grande distanciamento entre os elos que compõem a tríade cuidar-gerenciar-educar, essenciais para o sucesso da realização do cuidado.

Dessa forma, percebe-se que, como em todos os serviços de saúde e na hemoterapia especialmente, também é imprescindível atingir segurança e obter produtos e serviços com qualidade, haja vista a sua efetividade e segurança possuir grande influência no prognóstico do cliente.
No entanto, ainda que seja um procedimento comprovadamente eficaz, envolve riscos epidemiológicos $^{(3,13)}$. A efetivação da transfusão sanguínea de forma segura e qualificada, mediante padronização de processos e estabelecimento de normas e condutas, possibilita maior organização e controle no papel gerencial e assistencial do enfermeiro ${ }^{(5)}$.

Evidenciou-se, na fala de apenas um enfermeiro, a alusão ao gerenciamento de escalas de trabalho, porém não se pode conferir a tal instrumento o título formal de Dimensionamento da Equipe de enfermagem que, ao estabelecer os parâmetros mínimos para o cálculo dos profissionais de enfermagem das diferentes categorias, apresenta critérios e parâmetros necessários ao adequado dimensionamento ${ }^{(14)}$. Estudos ${ }^{(15-19)}$ trazem duas ponderações relevantes, quando se trata desse assunto na enfermagem. Primeiramente, que as organizações, por questões financeiro-administrativas, muitas vezes optam por manter em seus quadros um número ampliado de técnicos em enfermagem em detrimento dos profissionais de nível superior. Com isso, o foco reside na redução dos dispêndios financeiros da instituição. Outra ponderação relevante também apontada, é que o adequado dimensionamento da equipe de enfermagem incide diretamente na qualidade do cuidado prestado.

A Liderança contribui não apenas para o desenvolvimento do profissional que a desempenha, como também para a coordenação e articulação das atividades que envolvem a produção do cuidado $^{(6)}$. A capacidade de liderar consiste em uma das principais competências a serem desenvolvidas por enfermeiros no contexto hospitalar, pois as constantes alterações tecnológicas e a permanente necessidade de atenção da clientela requerem novas habilidades desses profissionais de saúde e ocasionam, como consequência imediata, transformações no seu processo de trabalho ${ }^{(20)}$.

Estudos sobre liderança e gerenciamento em enfermagem no ambiente hospitalar sinalizam que os enfermeiros apresentam perfil de liderança, ainda a exercem de forma moderada e, muitas vezes, com necessidade de aprimoramento na utilização de ferramentas e 
estratégias $^{(21-23)}$. Entende-se que essa fragilidade pode ser apontada como uma consequência da fragilidade do ensino e do baixo incentivo das instituições para o desenvolvimento permanente dos profissionais.

No ambiente hospitalar, as atividades executadas ocorrem em meio ao estresse, exigindo atenção e sinergia entre todos os envolvidos. Sendo assim, nesse contexto, é essencialmente importante a figura do enfermeiro na condição de líder, como motivador e intermediador das relações, para diminuir a sobrecarga da equipe e impactar positivamente na recuperação do cliente ${ }^{(24)}$.

Apesar de toda a fragilidade evidenciada na prática da liderança, neste estudo, os enfermeiros consideraram as estratégias de liderança no ambiente profissional como eficientes, passíveis de serem executadas e de aplicação necessária. Tais constatações fortalecem o entendimento da fragilidade do conhecimento teórico e prático de grande parte dos enfermeiros que exercem a função gerencial para aplicar, na práxis do exercício profissional, as habilidades e atitudes necessárias ao exercício eficaz da liderança.

O Planejamento do cuidado de enfermagem, como uma das ações de gerência do cuidado de enfermagem, faz parte do cotidiano laboral do enfermeiro, sendo necessário para o exercício da administração da assistência e para o desempenho da gerência de enfermagem ${ }^{(6)}$. Desse modo, entende-se que planejar é o ato de preparar-se para a execução de uma determinada ação ou tarefa, com vistas a atingir um determinado resultado que se espera no futuro. O exercício do planejamento de forma consciente e contínua e a sua correta aplicação é sinal de grandes possibilidades no sucesso da execução de tarefas ${ }^{(15)}$.

Nas falas dos participantes deste estudo, não foram evidenciadas ações que refletissem o planejamento do cuidado de enfermagem. Trechos das falas mostraram o "treinamento" como uma atribuição e necessidade da equipe de enfermagem no serviço pesquisado, o que converge com as ações de gerência do cuidado aqui investigadas, neste caso, o Treinamento/educação da equipe.
Os enfermeiros participantes demonstraram a necessidade de abordar mais extensamente o tema hemoterapia dentro do Hospital, tanto para eles quanto para sua equipe. Essa é uma demanda que necessita ser ouvida e levada para os gestores do serviço, para que esses profissionais tenham a capacitação necessária para a realização dos procedimentos inerentes à hematologia/hemoterapia, bem como o gerenciamento de sua equipe de trabalho.

Mais uma vez, pôde-se evidenciar as lacunas consequentes da formação desses profissionais, que se manifesta na pouca experiência, no caso dos recém-formados, ou, ainda, na falta de ações de educação permanente estruturadas nos serviços de saúde.

A ação Gestão de materiais ${ }^{(6)}$ não foi detectada nas falas dos participantes deste estudo, porém não se pode desconsiderar sua importância no contexto de qualquer serviço de saúde, haja vista que a sua ausência, em quantidade e qualidade adequadas, inviabiliza completamente a qualidade do cuidado. Dessa forma, a enfermagem deve não apenas participar do gerenciamento cotidiano desses recursos, utilizar tais recursos de forma planejada e consciente, juntamente com a sua equipe, como também relatar formalmente, aos setores competentes, qualquer inconformidade nesse sentido ${ }^{(25)}$.

A eficiência dos serviços prestados deve ser medida em constantes avaliações do resultado das ações previamente planejadas, de modo a contribuir para a promoção de serviços mais assertivos, eficazes e resolutivos ${ }^{(2,15)}$. Considere-se ainda a possível redução dos custos consequente da qualificação do trabalho executado. Como é comum em quase todo processo de trabalho, a Avaliação dos resultados das ações de enfermagem ${ }^{(6)}$, rotineiramente, é posta em lugares menos evidentes nos serviços de saúde, gerando, muitas vezes, resultados imprecisos e indesejados na conclusão do cuidado prestado.

A utilização dessa ferramenta, essencial à qualidade do cuidado prestado, só será de fato evidenciada por meio do fortalecimento da prática crítica-reflexiva entre os profissionais de enfermagem, em detrimento do foco na execução 
mecânica de procedimentos e do cumprimento de metas $^{(15)}$. Dessa forma, busca-se justificar a ausência de falas que confirmem a importância e a necessidade da aplicação de tal ação.

Como limitação deste estudo, pode-se destacar que se restringe a uma unidade apenas de serviço de saúde e possuiu um tempo reduzido para a sua realização. A ampliação desta pesquisa em outros serviços proporcionará condições para corroborar e reforçar os achados, bem como permitirá comparação com outras realidades institucionais e regionais.

Este estudo visa contribuir com a ampliação da prática da gerência do cuidado de enfermagem de maneira global, bem como com a consolidação dessa prática nos serviços de hemoterapia, proporcionando maior segurança e efetividade nas ações realizadas em tais serviços.

\section{Considerações Finais}

No presente estudo, evidenciou-se a prática das seguintes ações de gerência do cuidado de enfermagem entre os profissionais do serviço de hemoterapia: Coordenação do cuidado/Supervisão e Prestação do cuidado complexo. Ficou evidente que a maioria das ações de gerência do cuidado analisadas, tais como Planejamento do cuidado de enfermagem, Dimensionamento da equipe, Treinamento/educação da equipe, Liderança, Gestão de materiais e Avaliação dos resultados das ações de enfermagem, apresentaram-se fragilizadas ou ausentes na prática do gerente de enfermagem em hemoterapia.

Percebeu-se que as ações mais referidas pelos enfermeiros entrevistados destacaram aquelas mais comumente relacionadas às competências técnicas adquiridas pelo profissional no seu contexto de formação, enquanto as ações menos citadas relacionaram-se às competências e habilidades que comumente fazem parte das lacunas da educação formal.

Somente com o investimento contínuo e oportuno, por meio de medidas que proporcionem a adequada formação e a utilização de estratégias assertivas de educação permanente para o aperfeiçoamento profissional e operacional do enfermeiro e para a prática das atividades gerenciais que lhe competem, pode-se promover direta e positivamente a sua capacitação, para que ele possa conduzir a equipe de enfermagem em seu adequado processo de trabalho. Desse modo, será possibilitado o atendimento às necessidades do cliente em seu contexto holístico, contribuindo com a resolutividade da prestação dos cuidados e o alcance dos resultados esperados no processo de realização do cuidado.

\section{Colaborações:}

1 - concepção, projeto, análise e interpretação dos dados: Hilma Nazaré Mendes Bezerra;

2 - redação do artigo e revisão crítica relevante do conteúdo intelectual: Hilma Nazaré Mendes Bezerra, Aurilívia Carolinne Lima Barros, Rosana Amora Ascari, Thaís Cristina Flexa Souza e Jouhanna do Carmo Menegaz;

3 - aprovação final da versão a ser publicada: Jouhanna do Carmo Menegaz.

\section{Referências}

1. Brasil. Ministério da Saúde. Portaria MS/GM n. 158, de 4 de fevereiro de 2016. Redefine o regulamento técnico de procedimentos hemoterápicos [Internet]. Brasília (DF); 2016 [cited 2020 Sep 6]. Available from: https://bvsms.saude.gov.br/bvs/saudelegis/ $\mathrm{gm} / 2016 /$ prt0158_04_02_2016.html\#: :text= 2\%C2\%BA\%20O\%20regulamento\%20t\%C3\% A 9 cnico $\% 20$ de, $\% 2$ C $\% 20$ coleta $\% 2$ C $\% 20$ processamento $\% 2 \mathrm{C} \% 20$ estocagem $\% 2 \mathrm{C}$

2. Conselho Federal de Enfermagem. Resolução Cofen n. 629/2020. Aprova e Atualiza a Norma Técnica que dispõe sobre a Atuação de Enfermeiro e de Técnico de Enfermagem em Hemoterapia [Internet]. Brasília (DF); 2020 [cited 2020 Aug 20]. Available from: http://www.cofen. gov.br/wp-content/uploads/2020/03/RESOLU\% C3\%87\%C3\%83O-COFEN-N\%C2\%BA-629-2020.pdf

3. Agência Nacional de Vigilância Sanitária. Marco conceitual e Operacional de Hemovigilância: Guia para Hemovigilância no Brasil [Internet]. Brasília (DF); 2016 [cited 2020 Aug 20]. Available from: https://portaldeboaspraticas.iff.fiocruz.br/ biblioteca/marco-conceitual-e-operacional-dehemovigilancia-guia-para-hemovigilancia-nobrasil/ 
4. Forster F, Câmara AL, Moraes CLKM, Honório MT, Mattia D, Delacanal DL. Percepção dos enfermeiros quanto à assistência de enfermagem no processo transfusional. Enferm Foco. 2018;9(3):71-5. DOI: https://doi.org/10. 21675/2357-707X.2018.v9.n3.1509

5. Silva JDB, Sabino KCV, Brito LVBS, Silva AM, Costa ES, Brito ALS, et al. As hemotransfusões e atuação do enfermeiro nos procedimentos alternativos. Braz J Surg Clin Res [Internet]. 2018 [cited 2020 Aug 20];23(1):100-5. Available from: https://www.mastereditora.com.br/periodico/ 20180606_084138.pdf

6. Santos JLG, Pestana AL, Guerrero P, Meirelles BSH, Erdmann AL. Práticas de enfermeiros na gerência do cuidado em enfermagem e saúde: revisão integrativa. Rev bras enferm. 2013;66(2):257-63. DOI: https://doi.org/10.1590/S0034-716720130002 00016

7. Tong A, Sainsbury P, Craig J. Consolidated criteria for reporting qualitative research (COREQ): a 32-item checklist for interviews and focus groups. Int J Qual Health Care. 2007;19(6):349-57. DOI: https://doi.org/10.1093/intqhc/mzm042

8. Bardin L. Análise de conteúdo [Internet]. Lisboa: Edições 70; 2016 [cited 2020 Aug 20]. Available from: https://madmunifacs.files.wordpress.com/ 2016/08/anc3a1lise-de-contec3bado-laurencebardin.pdf

9. Paes LW. Atuação do enfermeiro na coordenação do cuidado: o caso da Catalunha [dissertação]. [Internet]. Rio de Janeiro: Escola Politécnica de Saúde Joaquim Venâncio, Fundação Oswaldo Cruz; 2019 [cited 2020 Aug 20]. Available from: https://www.arca.fiocruz.br/handle/icict/35588

10. Conselho Federal de Enfermagem. Resolução Cofen n. 511, de 31 de março de 2016. Aprova a Norma Técnica que dispõe sobre a atuação de Enfermeiros e Técnicos de Enfermagem em Hemoterapia: na coleta, armazenamento, administração, controle de qualidade, e outras atividades [Internet]. Brasília (DF); 2016 [cited 2020 Sep 6]. Available from: http://www.cofen.gov.br/ wp-content/uploads/2016/03/ANEXO-NORMAT\%C3\%89CNICA-ATUA\%C3\%87\%C3\%83ODE-ENFERMEIROS-E-T\%C3\%89CNICOS-DEENFERMAGEM-EM-HEMOTERAPIA-1.pdf

11. Agência Nacional de Vigilância Sanitária. Hemovigilância. Manual técnico de Hemovigilância - Investigação das reações transfusionais imediatas e tardias não infecciosas [Internet].
Brasília (DF); 2007 Nov [cited 2020 Aug 18]. Available from: http://www.cvs.saude.sp.gov.br/ zip/manual_tecnico_hemovigilancia_08112007.pdf

12. Agência Nacional de Vigilância Sanitária. Resolução RDC n. 51, de 29 de setembro de 2014. Dispõe sobre a Rede Sentinela para o Sistema Nacional de Vigilância Sanitária [Internet]. Brasília (DF); 2014 [cited 2020 Aug 18]. Available from: http://bvsms.saude.gov.br/bvs/saudelegis/ anvisa/2014/rdc0051_29_09_2014.html

13. Amaral JHS, Nunes RLS, Rodrigues LMS, Braz MR, Balbino CM, Silvino ZR. Hemoterapia: um desafio no cotidiano da equipe de enfermagem. Rev enferm UFPE on line. 2016;10 (Supl 6):4820-7. DOI: 10.5205/reuol.8200-718303-SM.1006sup201614

14. Conselho Federal de Enfermagem. Resolução Cofen n. 543, de 12 de maio de 2017. Estabelece os parâmetros para dimensionar o quantitativo de profissionais das diferentes categorias de enfermagem para os serviços/locais em que são realizadas atividades de enfermagem [Internet]. Brasília DF); 2017 [cited 2020 Sep 6]. Available from: http://www.cofen.gov.br/resolucao-cofen5432017_51440.html

15. Oliveira SA, Almeida ML, Santos MF, Zilly A, Peres AM, Rocha FLR. Ferramentas gerenciais na prática de enfermeiros da atenção básica em saúde [Internet]. Rev Adm saúde. 2017 [cited 2020 Aug 26];7(69):1-20. Available from: https://www. researchgate.net/profile/Samuel_De_Oliveira5/ publication/321505144_Ferramentas_gerenciais_ na_pratica_de_enfermeiros_da_atencao_basica_ em_saude/links/5ab3c2f2aca272171003930c/ Ferramentas-gerenciais-na-pratica-de-enfermeirosda-atencao-basica-em-saude.pdf

16. Silva LC, Oliveira DAL, Santos ABR, Barbosa LMS, Araújo LG, Barboza MTV, et al. Dimensionamento de pessoal e sua interferência na qualidade do cuidado. Rev enferm UFPE on line [Internet]. 2019 [cited 2020 Aug 20];13(1):491-8. Available from: https://periodicos. ufpe.br/revistas/revistaenfermagem/article/ viewFile/236551/31368\#: : :text=Conclus\% C3\%A30\%3A\%20pode\%2Dse\%20considerar\% 20que, de $\% 20$ profissionais $\% 20$ para $\% 20$ assisti $\%$ 2Dlos

17. Santos TA, Santos JNP, Lima SS, Marques DRS. Revisão Integrativa: o Planejamento Estratégico Situacional como um instrumento de gestão do enfermeiro [Internet]. Trabalho apresentado no 
International Nursing Congress; 2017 May 9-12; Aracaju: Unit; 2017 [cited 2020 Sep 6]. Available from: https://eventos.set.edu.br/cie/article/view $5562 / 2102$

18. Siqueira LDC, Santos MC, Calmon ITS, Siqueira Junior PC. Dimensionamento de profissionais de enfermagem da clínica médica de um hospital universitário. Enferm Foco. 2019;10(4):35-40. DOI: https://doi.org/10.21675/ 2357-707X.2019.v10.n4.2179

19. Grebinski ATKG, Biederman FA, Berte C, Barreto GMS, Oliveira JLC, Santos EB. Carga de trabalho e dimensionamento de pessoal de enfermagem em terapia intensiva neonatal. Enferm Foco. 2019;10(1):257-63. DOI: https://doi. org/10.21675/2357-707X.2019.v10.n1.1468

20. Balsanelli AP, Cunha ICKO. Liderança ideal e real dos enfermeiros de unidade de terapia intensiva em hospitais privados e públicos. Rev Cogitare Enferm. 2016;21(1):1-7. DOI: http://dx.doi. org/10.5380/ce.v21i4.42129

21. Martins RF, Gama JC, Carvalho ACR, Silva ME, Porto FR, Marta CB, et al. Gerenciamento e liderança em enfermagem: desafios e propostas de enfermeiros intensivistas. Saúde Coletiva (Barueri) [Internet]. 2020 [cited 2020 Aug 20];9(49):1488-93. Available from: http://www. revistas.mpmcomunicacao.com.br/index.php/ saudecoletiva/article/view/113
22. Amestoy SC, Trindade LL, Silva GTR, Santos BP, Reis VRSS, Ferreira VB. Liderança na enfermagem: do ensino ao exercício no ambiente hospitalar. Esc Anna Nery. 2017;21(4):e20160276. DOI: http:// dx.doi.org/10.1590/2177-9465-ean-2016-0276

23. Silva VLS, Camelo SHH, Soares MI, Resck ZMR, Chaves LDP, Santos FC, et al. Práticas de liderança em enfermagem hospitalar: uma self de enfermeiros gestores. Rev esc enferm USP. 2017;51:e03206. DOI: http://dx.doi.org/10.1590/ s1980-220x2016099503206

24. Melo MV. Estresse dos profissionais de saúde nas unidades hospitalares de atendimento em urgência e emergência [Internet]. Cadernos de Graduação - Ciências Biológicas e da Saúde Facipe. 2013 [cited 2020 Aug 20];1(2):35-42. Available from: file:///C:/Users/Usuario/Downloads/1200Texto\%20do\%20artigo-3740-1-10-20131113\%20(2).pdf

25. Ventura PFEV, Freire EMR, Alves M. Participação do enfermeiro na gestão de recursos hospitalares. Rev G\&amp;S [internet]. 2016 [cited 2020 Aug 20];7(1):126-47. Available from: https://periodicos. unb.br/index.php/rgs/article/view/3398

Recebido: 16 de junho de 2021 Aprovado: 9 de dezembro de 2021 Publicado: 26 de janeiro de 2022

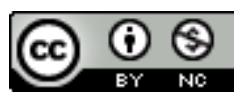

A Revista Baiana de Enfermagem utiliza a Licença Creative Commons - Atribuição-NãoComercial 4.0 Internacional. https://creativecommons.org/licenses/by-nc/4.0/ Este artigo é de acesso aberto distribuído sob os termos da Licença Creative Commons (CC BY-NC). Esta licença permite que outros remixem, adaptem e criem a partir do seu trabalho para fins não comerciais. Embora os novos trabalhos tenham de lhe atribuir o devido crédito e não possam ser usados para fins comerciais, os usuários não têm de licenciar esses trabalhos derivados sob os mesmos termos. 